\title{
Pathogenicity of indigenous Beauveria bassiana (Balsamo) against Ceratitis capitata Wiedemann (Diptera: Tephritidae) under laboratory conditions
}

\author{
Sabrina Chergui ${ }^{1^{*}} \mathbb{D}$, Khadidja Boudjemaa ${ }^{1}$, Abdelmadjid Benzehra ${ }^{1}$ and Ismail Karaca ${ }^{2}$ (D)
}

\begin{abstract}
Background: The Mediterranean fruit fly, Ceratitis capitata (Wiedemann) (Diptera: Tephritidae), is the major insect pest of fruit production worldwide. Chemical control of this pest has induced the selection of resistant medflies populations and negative environmental impact. In addition, the use of pesticides has become an obstacle to the fresh agricultural products exported to many countries that restrict pesticides residues. The aim of this study was to propose alternatives based on the use of microbiological products for the control of C. capitata.

Main body: The pathogenicity of the Turkish isolate Beauveria bassiana BMAUM M6-4 was evaluated against immature stages and adult of C. capitata under laboratory conditions at a concentration of $10^{7}$ conidia/ml via different routes of exposure. Conidial treatment was applied by spraying soil and filter paper against 3rd instar larvae and pupae. In contrast, the treatment was administered to adult males and females by contact and ingestion. The percentage of mortality was recorded on the 3rd, 7th, 12th and 15th day after inoculation. Obtained results showed that $B$. bassiana was very effective against the adult's fly, where males were more susceptible than females in both treatments (contact and ingestion). This isolate was tested against 3rd instar larvae. It achieved an infection rate of 33.33 and $43.5 \%$ of the larvae treated on filter paper and in the soil, respectively. Application of conidial preparations to soil reduced insect emergence and CAN represent a promising strategy for fruit fly integrated management (57.33 and 56.66\% emergence from the treatments in soil and filter paper).
\end{abstract}

Conclusion: Entomopathogenic fungi are promising as a biocontrol agent that can be used under different modes of ground application against larvae, prepupae and pupae, and/or as a bait spraying or contact against adults.

Keywords: Entomopathogenic fungi, Ceratitis capitata, Pathogenicity, Bioassay, Native isolate

\section{Background}

The Mediterranean fruit fly, Ceratitis capitata Wiedemann (Diptera: Tephritidae), is the main pest in the Mediterranean region attacking more than 300 hosts. It presents the greatest threat to the production and marketing of many fruit crops, mainly fruit crops. The pest overwinters as a pupa in the soil where it is a target for

\footnotetext{
* Correspondence: chergui.sabrina92@gmail.com

'Department of Agricultural and Forest Zoology, High National School of

Agronomy, Avenue Hassen Badi, El-Harrach, 16200 Algiers, Algeria

Full list of author information is available at the end of the article
}

many soil-dwelling organisms, including entomopathogenic fungi (EPF), particularly Ascomycetes, Beauveria spp. (Hypocreales: Cordycipitacea), and Metarhizium spp. (Hypocreales: Clavicipitaceae) (Vega et al. 2009; Rudeen et al. 2013). EPF can be isolated from plant tissues and soil. They use the soil as a habitat for longterm persistence when crops are not present in the field (Rudeen et al. 2013). Some of EPF species have a limited number of hosts, while the others have a wide range of hosts, for example, Beauveria bassiana (Bals.) Vuill. (Hypocreales: Cordycipitaceae) is an EPF that can infect 
a wide host range of over 700 insect species (De Faria and Wraight 2007).

EPF have received a considerable interest from scientists for their efficacy against crop pests and their biological control potential. The laboratory work serves as an initial indicator for the selection of fungal isolates as effective biocontrol agents in terms of their pathogenicity, specialization and ease of production and adaptation to environmental conditions (El-Husseini et al. 2018; Sayed et al. 2019).

The efficacy of EPF such as Metarhizium anisopliae and Beauveria bassiana on pupae and adults of C. capitata has been reported by several authors (Ekesi et al. 2005; Konstantopoulou and Mazomenos 2005). Despite the demonstrated virulence of these fungi against $C$. capitata, significant intraspecific variations in pathogen capacity among various strains and isolates of the same species have been observed, depending on the origin and initial host of the isolates (Castillo et al. 2000; QuesadaMoraga et al. 2006). Thus, selection of EPF isolates is one of the most important steps in a microbial control program, as the process determines which isolates are most virulent for the pest as well as their behavior with respect to relates to mortality, sporulation and the production of harmful organisms on an artificial culture medium (Rohde et al. 2006).

The indigenous Turkish isolate of $B$. bassiana BMAUM M6-4 was determined to cause infection to early and late instar larvae of the pine processionary Thaumetopoea wilkinsoni, (Lepidoptera: Notodontidae) (Gök et al. 2018) and larvae of Spodoptera littoralis (Lepidoptera : Noctuidae) (Cirbın et al. 2017). Therefore, this study aimed to evaluate the virulence of this isolate against larvae, pupae (newly formed and older) and adults of Ceratitis capitata in the laboratory via different routes of exposure.

\section{Materials and methods Insect rearing}

The rearing of C. capitata was carried out at the Department of Plant Protection, Suleyman Demirel University, at $25 \pm 1{ }^{\circ} \mathrm{C}, 65 \% \mathrm{RH}$, under 14:10 (L:D) photoperiod. Larvae were reared in sterile Petri dishes containing artificial diet: water $(56 \mathrm{ml})$, sugar $(12 \mathrm{~g}), \mathrm{Hcl}(0.3 \mathrm{ml})$, wheat germ $(4 \mathrm{~g})$, yeast $(3 \mathrm{~g})$, benzoic acid $(0.3 \mathrm{~g})$, bran $(23 \mathrm{~g})$. Adults were provided by water and a solid diet consisting of sucrose and yeast.

\section{Fungal isolate}

The EPF Beauveria bassiana BAUM M6-4, used in the following bioassays, was previously isolated from a soil sample obtained from an agricultural land in Isparta Province, Turkey (Baydar et al. 2016).

\section{Pathogenicity bioassays}

The experiments were conducted under the controlled conditions of $25 \pm 1{ }^{\circ} \mathrm{C}, 62 \pm 5 \% \mathrm{RH}$ and a photoperiod of 16:8 (L:D). Adults of C. capitata were maintained in cages and provided with diet for 5 days before using them for bioassays. The pupae and prepupae (3rd instar larvae) used in the experiments were obtained from the artificial rearing. One- and 6-day-old pupae were used for the bioassay. Fungal suspension at a concentration of $10^{7}$ conidia/ml was tested and mortality rates were assessed at 3, 7, 12, and 15 days after treatment. Each treatment was performed in 4 replicates (15 individuals each), with a total of 60 individuals.

The soil tested, was obtained from a peach orchard in Isparta. The soil was sieved and sterilized before use. Soil's choice was close to the natural control conditions of this pest in agricultural areas. The soil was used as an incubation substrate or as a self-inoculation device of prepupae and pupae.

\section{Exposure of 3rd larval instar to conidial suspension Essay on filter paper}

A group of 15 3rd instar larvae was placed on a filter paper in a sterile Petri dish $(9 \mathrm{~cm}$ in diameter) after spraying it with a volume of $3 \mathrm{ml}$ of conidial suspension at concentration of $10^{7}$ conidia $/ \mathrm{ml}$. The filter paper of the control group was sprayed with $3 \mathrm{ml}$ of distilled water and $0.1 \%$ Tween 80 .

\section{Soil bioassay}

A group of 15 3rd instar larvae were placed in a plastic container $(10 \times 6 \times 3)$ containing $50 \mathrm{~g}$ of natural soil and sieved after spraying this soil with $3 \mathrm{ml}$ of conidial suspension. For the control treatments, the same volume with $0.1 \%$ Tween 80 used distilled in water. There were four replicates of 15 larvae $(N=60 /$ treatment).

In both tests, the number of dead larvae was counted and the pupae formed from the treated larvae were monitored until the emergence of the adult.

\section{Exposure of pupae to conidial suspension Essay on filter paper}

A group of 15 new formed pupae of C. capitata, aged 1 day old and old pupae (6 days old), was deposited on a filter paper in a Petri dish after spraying it with a volume of $3 \mathrm{ml}$ of the suspension of conidia. Filter paper at the control group was sprayed with the same volume of $0.1 \%$ Tween 80 was used in distilled water.

\section{Soil bioassay}

A group of 15 C. capitata pupae, aged 1 and 6 days, was placed in a box $(10 \times 6 \times 3)$ containing $50 \mathrm{~g}$ of natural soil and sieved after spraying this soil with $3 \mathrm{ml}$ of 
suspension of conidia. For control treatments, $0.1 \%$ Tween 80 was used in distilled water.

Each treatment was replicated 4 times, i.e., 4 replicate groups of 15 pupae (total $=60$ ). In both tests, pupae were monitored until adult emergence.

\section{Exposure of adults to conidial suspension Contact bioassay}

In this bioassay, 15 5-day-old male and female adults of C. capitata were placed in a box $(11 \times 11 \times 6)$. A filter paper was placed on the base of the box and sprayed with $3 \mathrm{ml}$ of the conidia suspension before transferring C. capitata adults into the box. While walking on the treated filter paper, the flies were contacted to the spores. Each box received soaked cotton and a small amount of food. For control treatments, $0.1 \%$ Tween 80 was used in the water.

Dead adults were removed and transferred to another sterile Petri dish filled with wet filter paper. After sealing with Parafilm, the Petri dishes were stored at $25{ }^{\circ} \mathrm{C}$ and monitored daily for fungal symptoms.

\section{Oral bioassay}

One milliliter of $B$. bassiana suspension at a concentration of $10^{7}$ conidia/ml was mixed with $1 \mathrm{ml}$ of nutrient preparation for adults and placed on a piece of cotton. Flies consume this diet within 2-3 days. A diet without conidia was provided to adults and used as controls.

\section{Statistical analysis}

Mortalities were corrected, using Abbott formula (Abbott 1925). $\mathrm{LT}_{50}$ values were calculated using Probit analysis (Finney 1971). All statistical analyses were performed using GraphPad Prism version 8.0.0 at the 0.05 level of significance.

\section{Results and discussion}

Pathogenicity against 3rd larval instar

Data presented in Fig. 1 show the susceptibility of 3rd instar C. capitata larvae to conidial treatment. Mean mortality rate of larvae was $55.03 \%$ in filter paper treatment and $43.33 \%$ in the soil treatment. No mortality was recorded in the control. Non-significant difference was observed between the treatment of filter paper and that of the soil $(T=1.406 ; \mathrm{DF}=15 ; P>0.05)$. Treatment with $B$. bassiana was effective on both treatments.

Some treated larvae continued to pupate but a mortality rate occurred in the pupal stage. 41.20 and $44.97 \%$ emergence rates were recorded at the treatments in soil and filter paper, respectively, compared with $100 \%$ emergence in the control groups. The results revealed a significant difference between the emergence percentages of adults obtained in the control and in the treated larvae $(F=494.3, \mathrm{DF}=11, P<0.0001)$.

Obtained results are in contradiction with the results reported by Dimbi et al. (2003) who reported no effect for Metarhizium anisopliae and B. bassiana on larvae of $C$. capitata, C. rosa and C. cosyra, although they are highly virulent against the adult stage of these species. The results are in agreement with several studies reporting that the larval stage of the $C$. capitata was the most vulnerable to EPF (Ekesi et al. 2005; Quesada-Moraga et al. 2006).

\section{Pathogenicity against pupae}

Treatment of B. bassiana BAUM M6-4 caused mortality in $C$. capitata pupae, resulting in a decrease in the emergence rate of treated pupae than the untreated pupae $(F=$ 41.33, $\mathrm{DF}=11, P<0.0001)$. Figure 2 clearly shows that the treated pupae had a low emergence rate than the control. Obtained results revealed non-significant difference
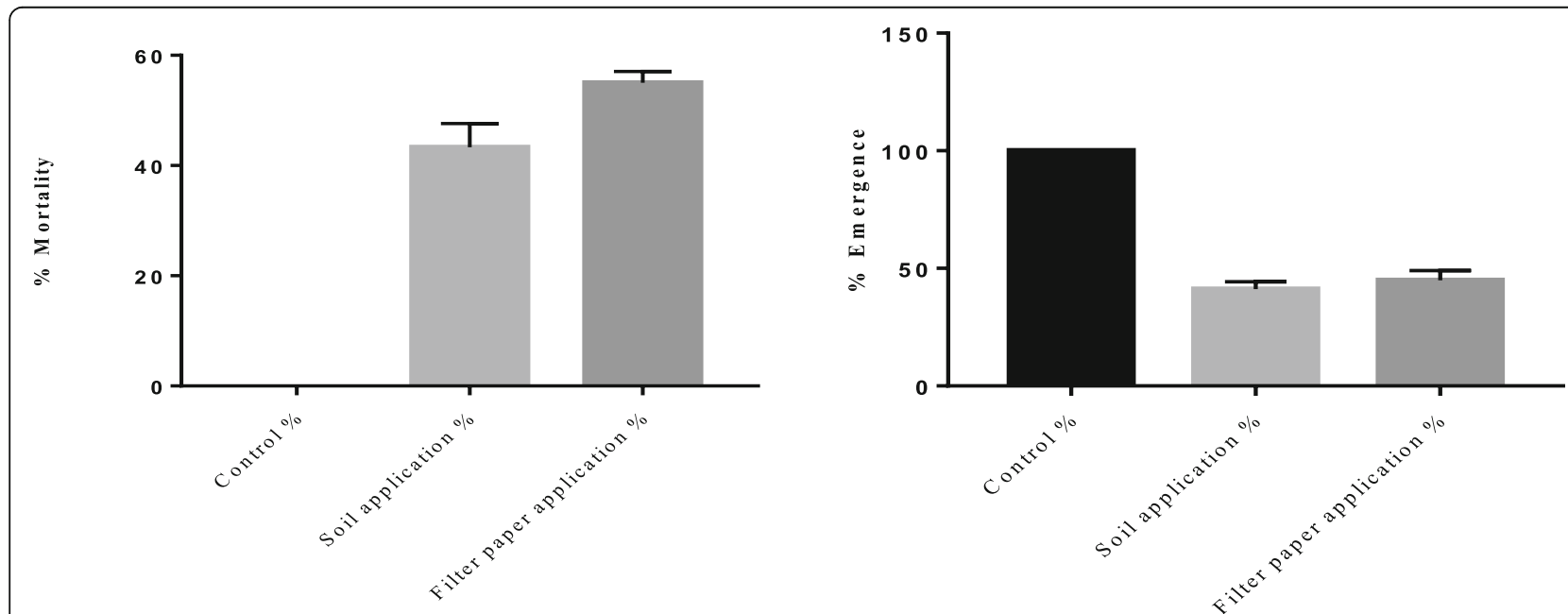

Fig. 1 Percentage mortality and emergence rate (Means+SE) of Ceratitis capitata larvae treated with Beauveria bassiana BMAUM M6-4 suspension (at $10^{7}$ conidia/ml) 


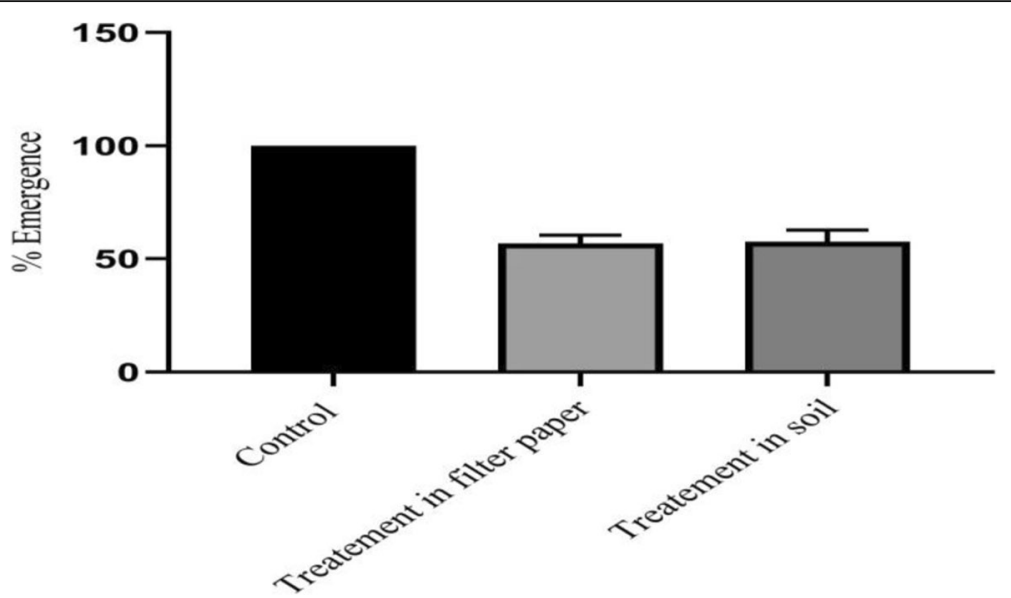

Fig. 2 Emergence rates (means + SE) of Ceratitis capitata pupae treated with Beauveria bassiana suspension at $10^{7}$ conidia/ml. a Soil application. b Filter paper application

between the two techniques of treatment (soil application and paper filter applications) $(T=0.9135 ; \mathrm{DF}=7 ; P>0.05)$.

Infection with the $B$. bassiana BAUM M6-4 against $C$. capitata pupae caused a significant reduction in emergence rate and number of adults (56.66 and 57.33\% emergence in filer paper and soil application, respectively, compared with $100 \%$ emergence in the control groups). This treatment contributed in reducing the population from one generation to the next. The $B$. bassiana BMAUM M6-4 isolate produced a dense condition on the treated pupae. A similar trend was reported by (Ekesi et al. 2005).

The results obtained in this study are consistent with those found by Mahmoud (2009) who tested Bactrocera zonata (Diptera: Tephritidae) but did not differ from those found by Quesada-Moraga et al. (2006) who reported mortality rates of $C$. capitata pupae to $B$. bassiana isolates ranging from 14 to $95.5 \%$. Previously, Lezama-Gutiérrez et al. (2000) reported that $M$. anisopliae reduced adult emergence by 33 and 49\% depending on soil type and about 37.9 to $98.75 \%$ mortality in Anastrepha ludens (Diptera: Tephritidae) larvae and puape, respectively. In another work, Alves et al. (2004) found that a high concentration of conidia $\left(1 \times 10^{8} \mathrm{co}-\right.$ nidia/ml) of $B$. bassiana and $M$. anisopliae was necessary to prevent the emergence of $C$. capitata.

Obtained results clearly showed that newly formed pupae ( 1 day old) were more susceptible to infection with EPF than the 6-day-old pupae in both the filter paper $(T=3.576$; $\mathrm{DF}=3 ; P<0.05)$ and soil tests $(T=$ 7.565; $\mathrm{DF}=3 ; P<0.05$ ) (Fig. 3). $46.67 \%$ emergence in larvae aged 1 day old was recorded in the soil treatment and $48.33 \%$ in the filter paper treatment against 65 and $68 \%$ emergence in the 6-day-old pupae in the 2 treatments, respectively.

Ekesi et al. (2002) evaluated the pathogenicity of $M$. anisopliae against pupae of 3 Tephritid species, including $C$. capitata, and found that pupal susceptibility
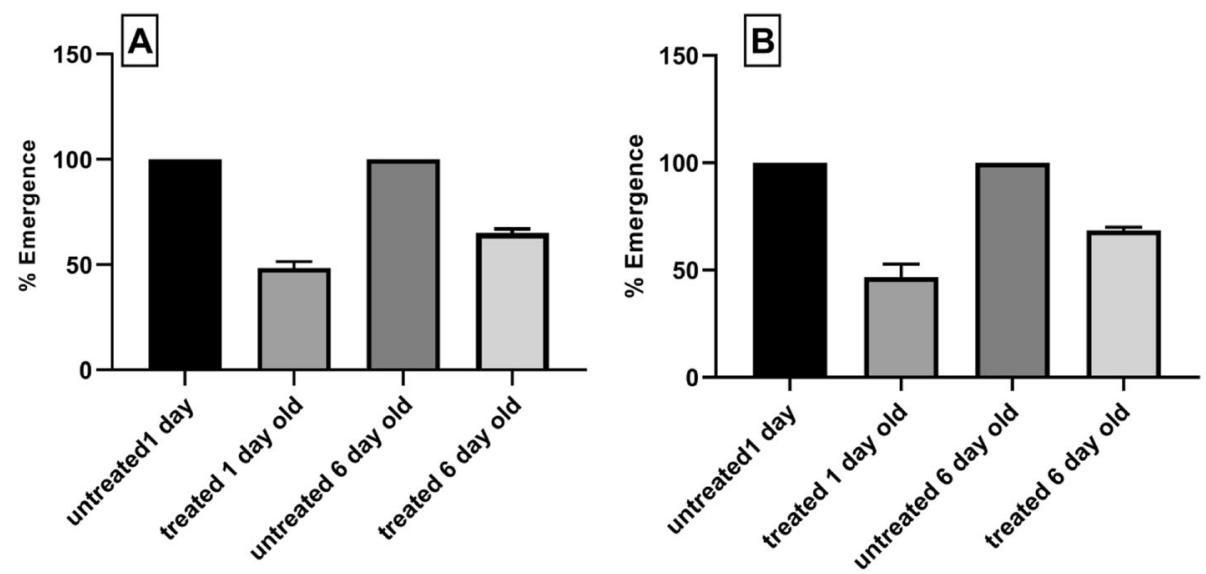

Fig. 3 Mortality percentage (means + SE) of Ceratitis capitata pupae treated with Beauveria bassiana suspension at $10^{7}$ conidia/ml 
decreased with increasing pupal age. In a recent study, Oreste et al. (2015) studied the interaction between age of puparia (at 2, 4, and 6 days old) and fungal strains. They addressed both the emergence of $C$. capitata adults and the mortality of pupae. They found that the fungal treatments of different $B$. bassiana strains were high on 2-day-old puparia (49.16 and $51.33 \%$ of mycosed puparia for ATCC 74040 and AL1 strain, respectively), while the rate of mycoses was low and ranged between 39 and $27.16 \%$, when fungal treatments were performed on 4and 6-day-old puparia. According to Beris et al. (2013), the highest susceptibility of young pupae can be attributed to their softer cuticle.

It was recorded that adults obtained from treated pupae had a high mortality than the control. Adult mortality in the treatments was $14.29 \%$ in the soil treatment and $22.22 \%$ in the filter paper treatment, in addition uncompleted emergence was also observed. Ekesi et al. (2002) cleared that the post-adult fungal infection was associated with the penetration of the hypha through the puparia integument in the body of the adult before emergence.

\section{Pathogenicity against adults}

The virulence of $B$. bassiana BMAUM M6-4 against adults of $C$. capitata was estimated 4 times after treatment. The results presented in Fig. 4 show the effect of oral and contact treatment on male and female adult mortality. No mortality was observed at the control for both sexes.

The average adult mortality of $C$. capitata after contact treatment with conidial suspension of $B$. bassiana strain BMAUM M6-4 reached $51.67 \%$ of adult females and $71.67 \%$ of adult males. A highly significant difference in mean mortality between males and females of $C$. capitata treated as contact method with conidial suspension of B. bassiana BAUM M6-4 at $10^{7}$ conidia/ml was found $(T=2.132$; $\mathrm{DF}=30 ; P<0.05)$. As well, similar results were estimated between males and females of $C$. capitata treated with oral method $(T=2.637 ; \mathrm{DF}=30$; $P<0.05)$. The average adult mortality of $C$. capitata after feeding a mixture of conidial suspension and artificial food for $48 \mathrm{~h}$ was higher in males (86.33\%) than in females (68.33\%). Obtained data revealed a significant difference between the two modes of application $(T=2.158$; $\mathrm{DF}=$ $30 ; P<0.05)$. The mortality induced by ingestion was significantly higher than that caused by contact. $73.33 \%$ and $86.33 \%$ of mortality were recorded toward adult males and females, respectively. Indeed, treated females by ingestion were more susceptible to infection $(68.33 \%$ mortality 15 days post-treatment) than those treated by contact (51.67\%) $(T=2.853$; DF $=30 ; P<0.05)$. Similary, males treated by ingestion were more susceptible to infection (68.33\% mortality) than those treated by contact $(73.33 \%)$ $(T=2.137 ; \mathrm{DF}=30 ; P<0.05)$.

Dead individuals of $C$. capitata, covered with a white mycelium characteristic of the fungus B. bassiana (Fig. 5). The average percentage of dead treated adults, which developed mycelium was $100 \%$.

In contact bioassay, on the 3rd day of treatment, mortality rate was $1.67 \%$ for females and $6.67 \%$ for males $(T=$ $1.567 ; \mathrm{DF}=6 ; P>0.05)$. On the 7th day, mortality became $10 \%$ for females and $33.33 \%$ for males $(T=7.00$; $\mathrm{DF}=6$; $P<0.05)$. On 12 th day, mortality of females was $28.33 \%$ and $58.33 \%$ of males $(T=6.646$; $\mathrm{DF}=6 ; P<0.05)$. A maximum of 51.67 and $73.33 \%$ was recorded on the 15 th day of treatment for males and females, respectively $(T=$ 13.01; $\mathrm{DF}=6 ; P<0.05)$.

In oral bioassay, on the 3th day of treatment mortality was $10.33 \%$ for females and $26.67 \%$ for males $(T=8.671$; $\mathrm{DF}=3 ; P<0.05)$. On 7 th day, the fungus caused 35 and $53.33 \%$ mortality in females and males, respectively $(T=$ 5.745; $\mathrm{DF}=6 ; P<0.05)$. On 12th day, the rate became $61.67 \%$ in females and $83.33 \%$ in males $(T=5.811$; $D=6$;

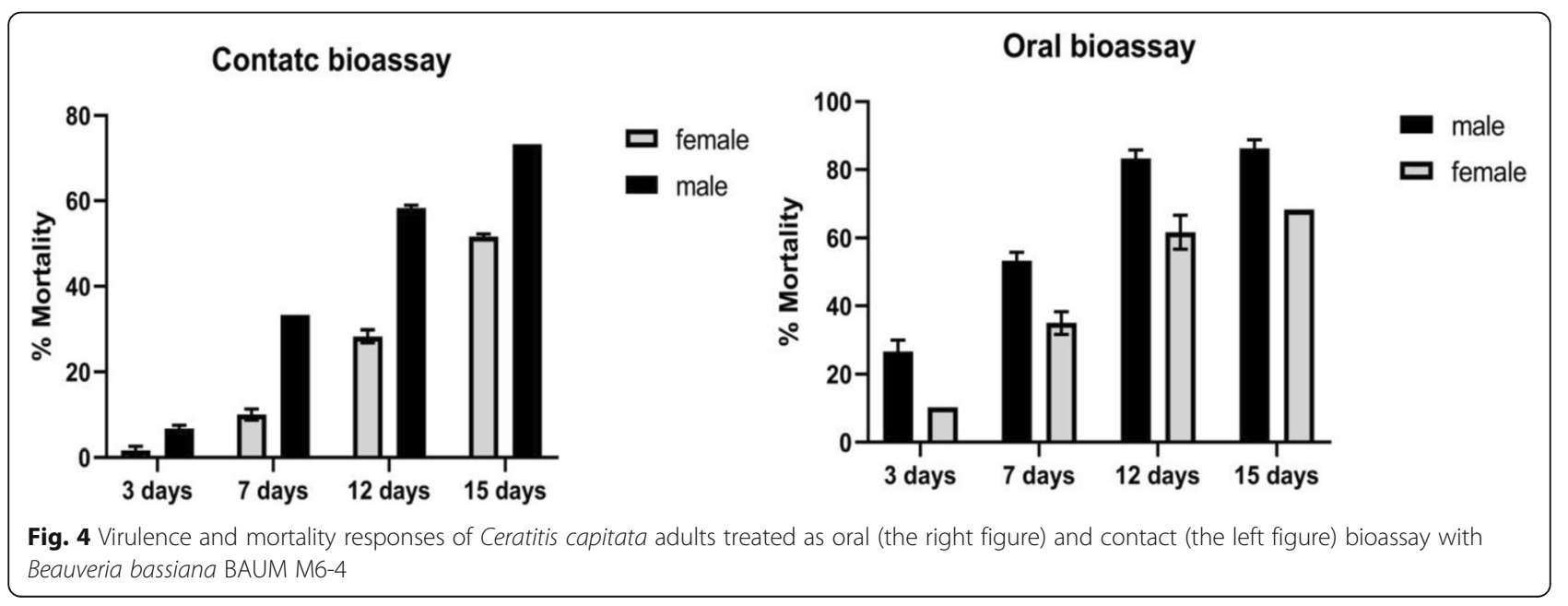




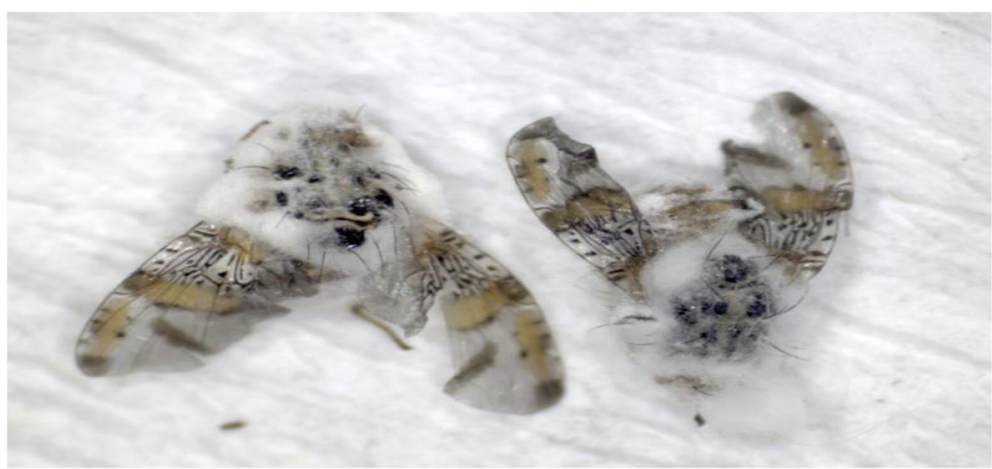

Fig. 5 Infection symptoms on adults of Ceratitis capitata induced by Beauvaria bassiana isolate BMAUM M6-4

$P<0.05)$. The maximum 86.33 and $68.33 \%$ were recorded on the 15th day of treatment for males and females of $C$. capitata, respectively $(T=11.01 ; \mathrm{DF}=6 ; P<0.05)$.

In oral treatment, Probit analysis of the time mortality response $\left(\mathrm{LT}_{50}\right)$ for adult females was 10.87 days. Whereas, chi square was 37.61. However, $\mathrm{LT}_{50}$ for male was 6.54 days. Whereas, chi-square was 45.59 . In contact treatment, the $\mathrm{LT}_{50}$ for adult females was 14.68 days. Whereas, chi-square was 27.28. For adult males $\mathrm{LT}_{50}$ for male was 10.54 days. Whereas, Chi square was 47.45. The slope values of EPF toward adults are presented in Table 1. The slopes of 5.13 and 4.95 were observed in oral bioassays, while they were 3.61 and 4.04 in contact bioassays. The $\mathrm{LT}_{50}$ values were significantly higher in contact bioassay than in contact bioassay $(F=8.569, P<$ $0.0001)$. The differences were non-significant in slopes $(F=1.598 ; P=0.26)$. However, the differences between intercepts were significant $(F=34.34 ; P<0.0001)$.

Obtained results agree with the findings Konstantopoulou and Mazomenos (2005) who reported a moderate pathogenicity of $B$. bassiana when tested as oral bioassay against Bactrocera oleae (Diptera: Tephritidae) adults (causing $62.6 \%$ mortality) and obtained $\mathrm{LT}_{50}$ values of 17 days. In the same publication, authors obtained $\mathrm{LT}_{50}$ values of 13.4 days for adults $C$. capitata inoculated as oral bioassay with $B$. bassiana.

The fungal treatment of $B$. bassiana BMAUM M6-4 against adults of $C$. capitata caused a cumulated mortality for both sexes, whereas the male mortality was higher than of the female in both treatments (oral and contact bioassay). This result suggests that the male adults were more susceptible than females which agree with (ElAkhdar and Ouda 2009 and Boudjelida and Soltani 2011). In addition, $\mathrm{LT}_{50}$ was shorter in adult males (6.54 and 10.54 days in oral and contact bioassay, respectively) than in adult females (10.87 and 14.68 days, for the same bioassays, respectively). For both tests (10.54 and 14.68 days in oral and contact bioassay, respectively), that confirms the susceptibility of males to infection with EPF than females. Mahmoud (2009) reported $\mathrm{LT}_{50}$ of 14.67 days for $B$. oleae treated with $B$. bassiana as oral treatment, while it was 16.6 days in contact treatment.

Fly mortality changes with time, starting 3rd day after inoculation. The effect of the fungus $M$. anisopliae against the 4th larvae and the adults of $C$. capitata showed a high toxicity with a concentration-response manner with a mortality started from day 3 to day 6 confirmed the results of Yee and Lacey (2005) and Boudjelida and Soltani (2011).

\section{Conclusions}

The present study reported that the BMAUM M6-4 strain of $B$. bassiana had a notable efficacy against different stages of $C$. capitata. The fungus could be a promising biological control agent under different modes of applications as conidial application to the soil against pupae and 3rd instar larvae. Further research is needed to determine the efficacy of this isolate in the field to assess its actual contribution as a biological control agent against the fruit flies.

Table $1 \mathrm{LT}_{50}$ of adult males and females of Ceratitis capitata treated as oral and contact bioassay with Beauveria bassiana BAUM M6-4

\begin{tabular}{|c|c|c|c|c|c|}
\hline \multicolumn{2}{|c|}{ Mode of treatment } & \multirow{2}{*}{$\frac{\text { TL50 } \pm \text { SE (day) }}{14.68 \pm 0.27}$} & \multirow{2}{*}{$\begin{array}{l}\text { Intercept } \\
4.354\end{array}$} & \multirow{2}{*}{$\frac{\text { Slope } \pm \text { SE }}{3.614 \pm 0.42}$} & \multirow{2}{*}{$\frac{X^{2}(\mathrm{DF}=9)}{37.61}$} \\
\hline Contact bioassay & Female & & & & \\
\hline & Male & $10.54 \pm 0.24$ & 5.547 & $4.041 \pm 0.53$ & 45.59 \\
\hline \multirow[t]{2}{*}{ Oral bioassay } & Male & $6.54 \pm 0.20$ & 5.770 & $4.959 \pm 0.55$ & 47.45 \\
\hline & Female & $10.87 \pm 0.19$ & 7.636 & $5.130 \pm 0.7$ & 27.28 \\
\hline
\end{tabular}




\section{Abbreviations}

EPF: Entomopathogenic fungi; $L T_{50}$ : Median lethal time

\section{Acknowledgements}

Not applicable.

\section{Authors' contributions}

CS: carried out the experiment and wrote the manuscript. BK: revised the manuscript, corrected language. BA: conceived and planned the experiments. Kl: Analyzed data and supervised the work. All authors read and approved the final manuscript.

\section{Funding}

Not applicable. This work was not supported by any funding body.

\section{Availability of data and materials}

Not applicable

Ethics approval and consent to participate

Not applicable

\section{Consent for publication}

Not applicable.

\section{Competing interests}

The authors declare that they have no competing interests.

\section{Author details}

'Department of Agricultural and Forest Zoology, High National School of Agronomy, Avenue Hassen Badi, El-Harrach, 16200 Algiers, Algeria. ${ }^{2}$ Plant Protection Department, Suleyman Demirel University, Isparta, Turkey.

Received: 30 June 2020 Accepted: 7 October 2020

Published online: 14 October 2020

\section{References}

Abbott WS (1925) A method of computing the effectiveness of an insecticide. J Econ Entomol 18:265-267

Alves SB, Rossi LS, Walder JM, Vieira SA (2004) Avaliação de fungos entomopatogênicos para Ceratitis capitata. Manejo Integrado de Plagas y Agroecología (CATIE)(no. 72) p. 31-38

Baydar R, Güven Ö, Karaca I (2016) Occurrence of entomopathogenic fungi in agricultural soils from Isparta province in Turkey and their pathogenicity to Galleria mellonella (L.) (Lepidoptera: Pyralidae) larvae. Egypt J Biol Pest Control 26:323

Beris E, Papachristos D, Fytrou N, Antonatos S, Kontodimas D (2013) Pathogenicity of three entomopathogenic fungi on pupae and adults of the Mediterranean fruit fly, Ceratitis capitata (Diptera: Tephritidae). J Pest Sci 86: $275-284$

Boudjelida H, Soltani N (2011) Pathogenicity of Metarhizium anisopliae (Metsch) on Ceratitis capitata L. (Diptera: Tephritidae). Ann Biol Res 2:104-110

Castillo M-A, Moya P, Hernández E, Primo-Yufera E (2000) Susceptibility of Ceratitis capitata Wiedemann (Diptera: Tephritidae) to entomopathogenic fungi and their extracts. Biol Control 19:274-282

Cirbın I, Güven Ö, Karaca I (2017) Effects of entomopathogenic fungi on Spodoptera littoralis (Boisd.) (Lep: Noctuidae) larvae. Gazi Osman Paşa Üniv Ziraat Fak derg 34:159-165

De Faria MR, Wraight SP (2007) Mycoinsecticides and mycoacaricides: a comprehensive list with worldwide coverage and international classification of formulation types. Biol Control 43:237-256

Dimbi S, Maniania NK, Lux SA, Ekesi S, Mueke JK (2003) Pathogenicity of Metarhizium anisopliae (Metsch.) Sorokin and Beauveria bassiana (Balsamo) Vuillemin, to three adult fruit fly species: Ceratitis capitata (Weidemann), C. rosa var. fasciventris Karsch and C. cosyra (Walker) (Diptera: Tephritidae). Mycopathologia 156:375-382

Ekesi S, Maniania NK, Lux SA (2002) Mortality in three African tephritid fruit fly puparia and adults caused by the entomopathogenic fungi, Metarhizium anisopliae and Beauveria bassiana. Biocontrol Sci Technol 12:7-17

Ekesi S, Maniania NK, Mohamed SA, Lux SA (2005) Effect of soil application of different formulations of Metarhizium anisopliae on African tephritid fruit flies and their associated endoparasitoids. Biol Control 35:83-91
El-Akhdar EA, Ouda SM (2009) Pathogenicity of different fungal isolates to the adult stage of the Mediterranean fruit fly, Ceratitis capitata (Wiedmann). Egypt J Biol Pest Control 19:5-10

El-Husseini MM, El-Heneidy AH, Awadallah KT (2018) Natural enemies associated with some economic pests in Egyptian agro-ecosystems. Egypt J Biol Pest Cont. 28(1):78

Finney DJ (1971) Probit analysis, 3rd edn. Cambridge University Press, Cambridge

Gök SG, Güven Ö, Karaca I (2018) Entomopatojen Fungus Beauveria bassiana'nın Çam Keseböceği (Thaumetopoea wilkinsoni Tams)'nin Farklı Dönemlerine Etkileri. Türk Biyo Mücadele Derg 9:7-18

Konstantopoulou MA, Mazomenos BE (2005) Evaluation of Beauveria bassiana and $B$. brongniartii strains and four wild-type fungal species against adults of Bactrocera oleae and Ceratitis capitata. BioControl 50:293-305

Lezama-Gutiérrez R, la Luz AT, Molina-Ochoa J, Rebolledo-Dominguez O, Pescador AR, López-Edwards M, Aluja M (2000) Virulence of Metarhizium anisopliae (Deuteromycotina: Hyphomycetes) on Anastrepha ludens (Diptera: Tephritidae): laboratory and field trials. J Econ Entomol 93:1080-1084

Mahmoud MF (2009) Pathogenicity of three commercial products of entomopathogenic fungi, Beauveria bassiana, Metarhizum anisopilae and Lecanicillium lecanii against adults of olive fly, Bactrocera oleae (Gmelin)(Diptera: Tephritidae) in the laboratory. Plant Protect Sci 45:98-102

Oreste M, Baser N, Bubici G, Tarasco E (2015) Effects of Beauveria bassiana strains on the Ceratitis capitata-Psyttalia concolor system. Bull Insectol 68(2):365-272

Quesada-Moraga E, Ruiz-García A, Santiago-Álvarez C (2006) Laboratory evaluation of entomopathogenic fungi Beauveria bassiana and Metarhizium anisopliae against puparia and adults of Ceratitis capitata (Diptera: Tephritidae). J Econ Entomol 99:1955-1966

Rohde C, Alves LF, Neves P, Alves SB, Silva E, Almeida J (2006) Seleção de isolados de Beauveria bassiana (Bals.) Vuill. Metarhizium anisopliae (Metsch.) Sorok. contra o cascudinho Alphitobius diaperinus (Panzer)(Coleoptera: Tenebrionidae). Neotrop Entomol 35:231-240

Rudeen ML, Jaronski ST, Petzold-Maxwell JL, Gassmann AJ (2013) Entomopathogenic fungi in cornfields and their potential to manage larval western corn rootworm Diabrotica virgifera virgifera. J Invertebr Pathol 114 329-332

Sayed SM, Ali EF, Al-Otaibi SS (2019) Efficacy of indigenous entomopathogenic fungus, Beauveria bassiana (Balsamo) Vuillemin, isolates against the rose aphid, Macrosiphum rosae L. (Hemiptera : Aphididae) in rose production. Egypt J Biol Pest Cont 29(1):19

Vega FE, Goettel MS, Blackwell M, Chandler D, Jackson MA, Keller S et al (2009) Fungal entomopathogens: new insights on their ecology. Fungal Ecol. 2:149159

Yee WL, Lacey LA (2005) Mortality of different life stages of Rhagoletis indifferens (Diptera: Tephritidae) exposed to the Entomopathogenic Fungus Metarhizium anisopliae. J Entomol Sci 40:167-177

\section{Publisher's Note}

Springer Nature remains neutral with regard to jurisdictional claims in published maps and institutional affiliations.

\section{Submit your manuscript to a SpringerOpen ${ }^{\bullet}$ journal and benefit from:}

- Convenient online submission

- Rigorous peer review

- Open access: articles freely available online

- High visibility within the field

- Retaining the copyright to your article

Submit your next manuscript at $>$ springeropen.com 